\title{
Black holes in low-mass bulges and pseudobulges
}

\author{
N. Nowak ${ }^{1}$, R.P. Saglia ${ }^{1}$, J. Thomas ${ }^{2}$, P. Erwin ${ }^{1,2}$ and R. Bender ${ }^{1,2}$ \\ ${ }^{1}$ Max-Planck-Institut für extraterrestrische Physik, Giessenbachstrasse, 85748 Garching, \\ Germany \\ email: nnowak@mpe.mpg.de \\ ${ }^{2}$ Universitätssternwarte, Scheinerstrasse 1, 81679 Munich, Germany
}

\begin{abstract}
Over the past decade we have learned that probably all ellipticals and bulges contain a central supermassive black hole (SMBH). The mass of the SMBH correlates both with the mass of the bulge component (about $0.15 \%$ of the bulge mass) and with the velocity dispersion $\sigma$ of the bulge. We are investigating whether these relations remain valid or how they change when galaxies with pseudobulges, very low-mass bulges or bulgeless galaxies are considered. Studying SMBH relations for both classical bulges and pseudobulges can reveal the importance of different growing mechanisms (mergers vs. secular evolution) for the evolution of SMBHs. Low-mass classical bulges and bulgeless galaxies may harbour seed black holes in their earliest evolutionary stages, and studying them is of paramount importance for understanding the link between bulge evolution and black hole growth.
\end{abstract}

Keywords. galaxies: kinematics and dynamics, galaxies: bulges

\section{Introduction}

It is well established that all galaxies with a massive bulge component harbour a central supermassive black hole (SMBH). The mass of this SMBH $M_{\bullet}$ correlates with bulge properties such as the bulge mass and the velocity dispersion $\sigma$. Our knowledge of the behaviour of these correlations is mainly based on studies of massive ellipticals or earlytype spiral galaxies with a massive classical bulge component, but is rather poor for other types of galaxies and bulges. Currently the low- $\sigma$ end $(\sigma<120 \mathrm{~km} / \mathrm{s})$ is insufficiently constrained, with very few stellar or gas dynamical SMBH mass measurements. Even more unclear is the situation for galaxies that do not have a bulge at all. A few studies indicate that low-mass black holes may be present in bulgeless galaxies, but possible correlations with host galaxy properties are unclear.

If the growth of SMBHs is as tightly connected to the growth of the surrounding bulge as the $M_{\bullet}-\sigma$ relation suggests, the question arises whether SMBHs in pseudobulges obey the same correlations as SMBHs in classical bulges. Pseudobulges are the result of secular evolution. They have disclike characteristics, are flattened, often show spiral structure or nuclear bars and are rotation dominated. Real systems can contain both a classical and a pseudobulge component at the same time. Two of the three SMBHs found in pseudobulges are actually located in such compound systems (NGC 2787 and NGC 3384). Based on only these two examples it is not possible to conclude whether their masses scale with classical bulges only, or also with pseudobulges (Erwin et al. 2007).

\section{Observations and first results}

The goal of our study is to measure black hole masses for a larger number of low- $\sigma$ galaxies and to investigate if and how the $M_{\bullet}-\sigma$ relation changes when only pseudobulges 
Table 1. Galaxies observed with SINFONI and their distance $D$, velocity dispersion $\sigma$, sphere of influence $d_{\text {soi }}$ and spatial resolution $(\mathrm{FWHM})$

\begin{tabular}{lcccccc}
\hline Galaxy & $D(\mathrm{Mpc})$ & $\sigma(\mathrm{km} / \mathrm{s})$ & $d_{\text {soi }}\left(^{\prime \prime}\right)$ & resolution $\left(^{\prime \prime}\right)$ & bulge type & AO mode \\
\hline NGC 1398 & 18 & 200 & 0.34 & $0.19 \ldots 0.32$ & pseudo & NGS \\
NGC 3368 & 10 & 128 & 0.22 & $0.15 \ldots 0.25$ & pseudo & LGS \\
NGC 3627 & 10 & 115 & 0.19 & $0.15 / 0.088$ & pseudo & LGS \\
NGC 3489 & 12 & 105 & 0.12 & 0.08 & class. & NGS \\
NGC 4486a & 16 & 111 & 0.13 & 0.10 & class. & NGS \\
NGC 5102 & 4 & 65 & 0.10 & $0.12 / 0.07$ & class. & NGS \\
NGC 1316 & 19 & 220 & 0.44 & $0.12 / 0.09$ & class. & NGS \\
\hline
\end{tabular}

are considered. In inactive galaxies, the evidence for the existence of black holes and their masses comes from gravitational effects on the dynamics of stars or gas inside the black hole's sphere of influence. Since the radius of the sphere of influence scales with $M_{\bullet}$, high resolution observations are needed to detect $\mathrm{SMBH}$ in the low-mass regime. We therefore use the the integral-field spectrograph SINFONI (Eisenhauer et al. 2003, Bonnet et al. 2004), which can deliver diffraction-limited resolution thanks to the adaptive optics (AO) system, and is able to penetrate dust as it operates in the near-IR. We have observed seven galaxies in the $K$ band so far, two with the recently commissioned laser guide star (LGS) PARSEC (Rabien et al. 2004), the rest in natural guide star (NGS) mode. Table 1 gives an overview of the observations and the achieved resolutions.

The stellar kinematics is extracted from the CO bandheads at $>2.29 \mu \mathrm{m}$ using the maximum penalized likelihood method of Gebhardt et al. (2000) with a number of latetype stars observed with SINFONI as templates. The dynamical modelling is based on the Schwarzschild superposition code of Thomas et al. (2004). After obtaining the luminosity profile from high-resolution imaging, orbit libraries are constructed in a number of gravitational potentials, each of them calculated with a different black hole mass $M_{\bullet}$ and mass-to-light ratio $\Upsilon$. The best-fitting parameters then follow from a $\chi^{2}$-analysis.

The first result of our study is the detection of a SMBH in the low-luminosity elliptical galaxy NGC 4486a in the Virgo cluster. The bright star $\approx 2.5^{\prime \prime}$ away from the centre was used as a natural guide star for $\mathrm{AO}$ correction and offered a spatial resolution of $\approx 0.1^{\prime \prime}$. A black hole of mass $M_{\bullet}=1.25_{-0.79}^{+0.75} \times 10^{7} \mathrm{M}_{\odot}(90 \%$ C.L.) was found (Nowak et al. 2007 ), in perfect agreement with the predictions of the $M_{\bullet}-\sigma$ relation of Tremaine $e t$ al. (2002). The analysis of the remaining galaxies is under way. Particularly interesting is the pseudobulge galaxy NGC 3368, which shows a stellar $\sigma$ drop and two infalling, counterrotating clouds of $\mathrm{H}_{2}$ in the centre. Observations of more galaxies are planned to build a statistically significant sample of pseudobulges and low-mass bulges.

\section{References}

Erwin, P., et al. 2007, in prep.

Eisenhauer, F., et al. 2003, in: M. Iye, A. Moorwood (eds.), Instrument Design and Performance for Optical/Infrared Ground-based Telescopes Vol. 4841 of Proc. SPIE, p. 1548

Bonnet, H., et al. 2004, ESO Messenger 117, 17

Rabien, S., et al. 2004, in: D. Bonaccini, B. Ellerbroek, R. Ragazzoni (eds.), Advancements in Adaptive Optics, Vol. 5490 of Proc. SPIE, p. 981

Gebhardt, K., et al. 2000, AJ 119, 1157

Thomas, J., et al. 2004, MNRAS 353, 391

Nowak, N., et al. 2007, MNRAS 379, 909

Tremaine, S., et al. 2002, ApJ 574, 740 